\title{
rLj-RGD3, a Novel Three-RGD-Motif-Containing Recombinant Protein from Lampetra japonica, Protects PC12 Cells from Injury Induced by Oxygen-Glucose Deprivation and Reperfusion
}

\author{
Li Lv, ${ }^{1,2}$ Qian Lu, ${ }^{2,3}$ Fangyu Shao, ${ }^{2}$ Weiping Li, ${ }^{2}$ Qin Zhou, ${ }^{2}$ Jihong Wang, ${ }^{1}$ and Qingwei Li ${ }^{1}$ \\ ${ }^{1}$ School of Life Sciences, Liaoning Normal University, Dalian, Liaoning 116029, China \\ ${ }^{2}$ Department of Pharmacology, Dalian Medical University, Dalian, Liaoning 116044, China \\ ${ }^{3}$ College of Basic Medicine, Jilin Medical University, Jilin, Jilin 132013, China \\ Correspondence should be addressed to Jihong Wang; jihongwang999@hotmail.com and Qingwei Li; liqw@263.net
}

Received 22 March 2016; Accepted 3 July 2016

Academic Editor: Jane Hanrahan

Copyright (C) $2016 \mathrm{Li} \mathrm{Lv}$ et al. This is an open access article distributed under the Creative Commons Attribution License, which permits unrestricted use, distribution, and reproduction in any medium, provided the original work is properly cited.

\begin{abstract}
rLj-RGD3 is a $14.5 \mathrm{kDa}$ recombinant protein with $3 \mathrm{RGD}$ (Arg-Gly-Asp) motifs from the salivary gland secretions of Lampetra japonica, which is a histidine-rich and arginine-rich protein. Previous reports indicated that rLj-RGD3 has typical functions of RGD-toxin protein, such as platelet aggregation suppression tumour metastasis and angiogenesis inhibition. Because histidine and arginine have cerebral ischemia-reperfusion and neuroprotective functions, we investigated whether rLj-RGD3 has such activities and studied the mechanism. The effects of rLj-RGD3 on neuroprotection and antiapoptosis were determined. The expression level of focal adhesion kinase (FAK), p-FAK, Caspase-3, and Bcl-2 after oxygen-glucose deprivation and reperfusion (OGD-R) was examined. The viability of PC12 cells incubated with $\mathrm{rLj}$-RGD3 at high concentrations $(16 \mu \mathrm{mol} / \mathrm{L})$ increased significantly due to its ability to protect the cells from apoptosis after OGD-R-induced injury. Furthermore, rLj-RGD3 attenuated the damage due to OGD-R. Most of the PC12 cells were apoptotic after OGD-R. In contrast, the number of apoptotic PC12 cells was significantly decreased in the group treated with a high-dose of rLj-RGD3. In addition, $r L j-R G D 3$ activated FAK and p-FAK protein. rLj-RGD3 inhibited Caspase-3 and upregulated Bcl-2 protein expression in PC12 cells after OGD-R. The study provides the first evidence for neuroprotective effects of rLj-RGD3 in ischemic injury that may be partly mediated through inhibition of Caspase-3 and upregulation of $\mathrm{Bcl}-2$, FAK, and p-FAK protein expression.
\end{abstract}

\section{Introduction}

Ischemic stroke, commonly known as cerebral infarction, is a cerebral insufficiency caused by the brain tissue ischemia and hypoxia associated with the clinical syndrome. The main clinical manifestations include sensory loss, facial paralysis, ataxia, swallowing difficulties, speech problems, and visual disorders. Ischemic stroke has become the third largest cause of death in humans [1], and its incidence continues to increase yearly. Therefore, the development of drugs that provide effective protection from hypoxic-ischemic brain damage and inhibition of neuronal apoptosis is needed.

The integrin family of cell adhesion molecules primarily mediates the adhesion between cells and between the cells and the extracellular matrix (ECM), as well as inducing bidirectional signalling between the ECM and cells [2]. Integrin-mediated adhesion regulates many cell functions such as apoptosis [3], proliferation, adhesion, and migration [4], including lymphocyte homing. Currently, specific binding sites between extracellular matrix proteins and the integrin receptors have been identified, namely, the arginineglycine-aspartic sequence (Arg-Gly-Asp, RGD). A polypeptide containing the RGD sequence can recognise the integrin receptor and bind to it, thereby influencing cellular biological functions [5].

An increasing number of investigations have been committed to the development of RGD peptide-containing drugs. In recent years, a large number of studies have shown that 
RGD peptides can inhibit tumour cell metastasis, vascular endothelial cell growth, osteoclast activity, and platelet aggregation $[6,7]$.

$\mathrm{rLj}-\mathrm{RGD} 3$ is a $14.5 \mathrm{kDa}$ recombinant protein with $3 \mathrm{RGD}$ (Arg-Gly-Asp) motifs from the salivary gland secretions of Lampetra japonica, which is a histidine-rich and argininerich protein. Previous study results investigated that $\mathrm{rLj}$ RGD3 has typical functions of RGD-toxin protein, such as platelet aggregation suppression, tumour metastasis, and angiogenesis inhibition [8]. Because histidine and arginine have cerebral ischemia-reperfusion and neuroprotective functions, we investigated whether rLj-RGD3 has such activities on the focal cerebral ischemia and studied the mechanism, such as the role of FAK in the regulation of the integrin-PI3K/Akt pathway by rLj-RGD3 in the OGD-Rinduced PC12 cells damage model.

\section{Materials and Methods}

2.1. Materials. The recombinant plasmids pET23b-RGD3 are preserved in our laboratory. Histidine affinity chromatography columns (His. Bind Column) were purchased from Novagen, Tricine, and isopropyl $\beta$-D-1-thiogalactopyranoside (IPTG) were purchased from Amresco.

2.2. Extraction and Purification of the rLj-RGD3 Proteins. BL21 E. coli cells containing the recombinant plasmids pET23b-RGD3 were cultured until the cells reached the logarithmic phase of growth, which corresponded to an OD600 value (optical density value at $600 \mathrm{~nm}$ ) of 0.6 . Subsequently, isopropyl $\beta$-D-1-thiogalactopyranoside (IPTG) was added to a final concentration of $1 \mathrm{mM}$, and the cells were cultured at $30^{\circ} \mathrm{C}$ overnight to induce the expression of soluble $\mathrm{rLj}$ RGD3. The BL21 cells were then sonicated and centrifuged, and the target proteins were purified via histidine affinity chromatography according to the manufacture's protocol.

The purified $r L j-R G D 3$ were identified through TricineSDS PAGE in the presence of glycerol, which is commonly used to separate proteins with low molecular weights. The experimental procedure included gel preparation, sample loading, fixation, staining, and destaining until clear protein bands were visible.

2.3. Cell Culture. Normal PC12 cells were purchased from the Institute of Cell Biology, Chinese Academy of Sciences (Shanghai), and maintained in Dulbecco's Modified Eagle's Medium (DMEM, Sigma, USA) supplemented with 5\% heatinactivated foetal calf serum (FCS, Sigma, USA), 10\% heatinactivated horse serum, penicillin $(50 \mathrm{U} / \mathrm{mL})$, and streptomycin $(50 \mathrm{mg} / \mathrm{L})$. PC12 cells were cultured at $37^{\circ} \mathrm{C}$ in a humidified atmosphere of $5 \% \mathrm{CO}_{2}$ and $95 \%$ air. The cells were divided into six groups: the control group; the OGD-R model group; the rLj-RGD3 high-dose $(16 \mu \mathrm{mol} / \mathrm{L})$, mediumdose $(8 \mu \mathrm{mol} / \mathrm{L})$, and low-dose $(4 \mu \mathrm{mol} / \mathrm{L})$ groups; and the edaravone $(23 \mu \mathrm{mol} / \mathrm{L})$ positive control group.

2.4. Oxygen-Glucose Deprivation and Reperfusion. PC12 cells were subjected to OGD-R as described previously [9]. Briefly, the original medium was removed, and the cells were washed with Earle's balanced salt solution (EBSS) at pH 7.4 and placed in fresh glucose-free EBSS supplemented with $\mathrm{Na}_{2} \mathrm{~S}_{2} \mathrm{O}_{4}$. The culture dishes were then introduced in a mixture of $5 \% \mathrm{CO}_{2}$ and $95 \%$ air at $37^{\circ} \mathrm{C}$ for $2 \mathrm{~h}$, after which the medium was replaced with fresh DMEM for another $24 \mathrm{~h}$. The control culture was maintained in normal EBSS and incubated under the normal conditions. Edaravone was added to the culture 30 min before the OGD treatment.

2.5. Cell Viability. Cellular viability was evaluated using 2(4,5-dimethylthiazol-2-yl)-2,5-diphenyltetrazolium bromide (MTT; Sigma Corp., USA). The MTT reagent was dissolved in phosphate-buffered saline (PBS) and added to the culture at a final concentration of $0.5 \mathrm{mg} / \mathrm{mL}$. After an additional $4 \mathrm{~h}$ incubation at $37^{\circ} \mathrm{C}$, the media were carefully removed, and the triple fluid ( $10 \mathrm{~g}$ sodium dodecyl sulphonate, $5 \mathrm{~mL}$ of isobutanol, and a drop of hydrochloric acid, volume adjusted to $100 \mathrm{~mL}$ ) was added to each well. The OD was measured on a plate reader at $570 \mathrm{~nm}$ after $15 \mathrm{~h}$. The results are expressed as the percentages of the control.

2.6. LDH Activation Assays. Damage to the cells results in the release of lactate dehydrogenase (LDH). Therefore, the LDH activity in the supernatant fluid rises significantly. The total $\mathrm{LDH}$ activity in the supernatants was measured using the LDH Activity Assay Kit (Nanjing Jiancheng Corp., China) in accordance with the manufacturer's instructions. The results are expressed as $\mathrm{U} / \mathrm{g}$ protein.

2.7. Determination of Reduced GSH. GSH is an important free radical scavenger and antioxidant in vivo. It has antioxidant, antiaging, immunity-enhancing, and other physiological functions. The cells $\left(1 \times 10^{5} / \mathrm{mL}\right)$ were harvested after treatment with OGD for $2 \mathrm{~h}$ and reperfusion for $24 \mathrm{~h}$. The cells were washed twice using cold PBS. The concentration of GSH in the cells was determined using a commercial assay kit (Nanjing Jiancheng Corp., China) according to the manufacturer's instructions and expressed as g of GSH/L.

\subsection{Acridine Orange/Ethidium Bromide (AO/EB) Staining.} Sterilised coverslips were placed into 6-well plates, which were then placed in a laminar flow UV disinfection system overnight. Normal PC12 cells were plated into 6-well plates at a density of $1 \times 10^{5} / \mathrm{mL}$ cell. The model was performed as described above.

Acridine orange $(\mathrm{AO})$ can penetrate the cell membrane and bind to DNA, with the result that the cells appear green. In contrast, ethidium bromide (EB) only can access DNA through a damaged cell membrane, labelling the cells with an orange-red colour that is brighter than the green $\mathrm{AO}$ stain. AO and EB were each dissolved in $10 \mathrm{~mL}$ of PBS at a concentration of $10 \mathrm{mg} / \mathrm{mL}$. Equal volumes of the two solutions were mixed together before use. After a $24 \mathrm{~h}$ OGD$\mathrm{R}$ incubation, the coverslips were gently removed from the wells with tweezers, and $10 \mu \mathrm{L}$ of the $\mathrm{AO} / \mathrm{EB}$ mixture was applied. Then, the coverslip was inverted onto a glass slide. The images were acquired using fluorescence microscopy. 
2.9. Flow Cytometric Analysis. For the quantitative assessment of apoptosis, Annexin V-FITC and PI double-staining by flow cytometry were performed. Briefly, $5 \times 10^{6} / \mathrm{mL}$ PC12 cells were washed three times with cold PBS $\left(4^{\circ} \mathrm{C}\right)$ and resuspended in $300 \mu \mathrm{L}$ of binding buffer (BB). The cells were labelled according to the kit instructions (Beyotime Bio Co., Beijing, China). The cells were analysed immediately using flow cytometry. The signals from apoptotic cells are localised in the lower right quadrant of the resulting dot-plot graph [10].

2.10. Western Blot Analysis. Equal amounts of protein $(60 \mu \mathrm{g})$ were separated on $12 \%$ SDS-PAGE gels for detection of FAK and p-FAK and $15 \%$ gels for Caspase- 3 and $\mathrm{Bcl}-2$ protein levels and then transferred to nitrocellulose membranes. The membranes were then blocked with 5\% skim milk in PBS containing $0.1 \%$ Tween 20 (PBS-T). After washing with PBS$\mathrm{T}$, the membranes were probed overnight at $4^{\circ} \mathrm{C}$ with primary antibodies against FAK, p-FAK, Caspase-3, or Bcl-2 (all at a $1: 1000$ dilution). GAPDH was also detected with appropriate primary antibodies to ensure equal loading of cytoplasmic and nuclear proteins. Horseradish peroxidase-conjugated secondary antibodies (all at a 1:2000 dilution) were then applied to membranes, and the blots were developed with DAB. The expression level of each protein was determined by analysing the signal captured on the membrane using a gel imaging system.

2.11. Statistical Analyses. All values are expressed as the mean \pm SEM. Statistical comparisons were made using a one-way ANOVA test, followed by the Student-Newman-Keuls (SNK) test. In all cases, a difference was considered significant when $p$ was less than 0.05 . All statistical analyses were conducted using the SPSS16 statistical software package (SPSS, Inc., Chicago, IL, USA).

\section{Results}

3.1. Expression and Purification of $r L j-R G D 3$. $r L j-R G D 3$ was expressed as a soluble fusion protein in E. coli BL21 cells and purified via histidine affinity chromatography. The sequence of purified $\mathrm{rLj}$-RGD3 was confirmed by $\mathrm{NH} 2$-terminal amino acid sequencing. The protein was approximately $14.5 \mathrm{kDa}$ and migrated as a single band on a Tricine SDS-PAGE gel (Figure 1). The concentration of purified rLj-RGD3 was $15 \mu \mathrm{g} / \mu \mathrm{L}$.

The amino acid sequence of Lj-RGD3 contains 118 amino acids including 2 cysteines, 17 histidine, 17 arginine, and 20 threonine as well as 3 RGD motifs (Figure 2). The histidine-rich and arginine-rich characteristics of Lj-RGD3 suggested that it will have the functions of cerebral ischemiareperfusion and neuroprotection.

3.2. Effect of rLj-RGD3 on PC12 Cell Viability after OGD-R. The cell viability in the OGD-R-treated group was $45.0 \pm 2.4 \%$, whereas those in the rLj-RGD3-treated groups (at 16, 8 , and $4 \mu \mathrm{mol} / \mathrm{L})$ were $67.2 \pm 2.7 \%(p<0.01), 58.6 \pm 2.3 \%(p<$ $0.01)$, and $55.4 \pm 2.6 \%(p<0.01)$, respectively, compared

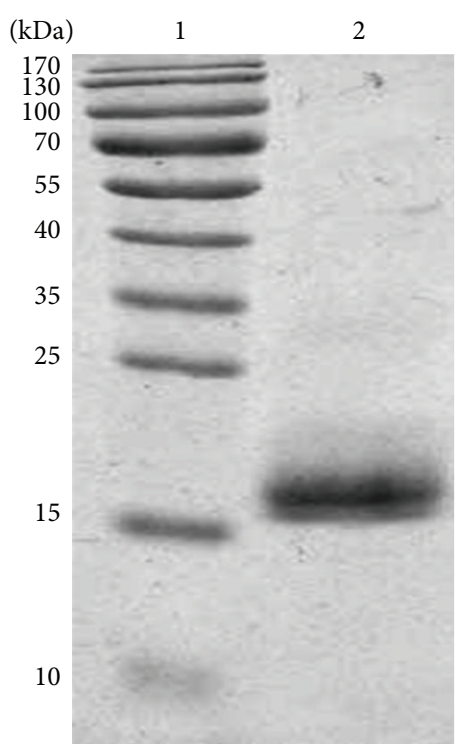

FIGURE 1: Tricine SDS-PAGE result of purified rLj-RGD3. Lane 1: protein marker; Lane 2: $14.5 \mathrm{kDa}$ of purified $\mathrm{rLj}$-RGD3.

\begin{tabular}{|lllllllllllllll|}
\hline M & S & T & F & I & N & G & N & T & Q & E & V & D & A & I \\
C & H & K & Q & N & Y & P & M & G & T & E & T & Q & G & D \\
T & R & G & D & T & R & T & H & T & E & T & Q & A & E & A \\
R & T & H & A & E & T & H & G & D & T & R & G & D & T & R \\
R & H & T & W & R & H & T & R & R & H & T & D & T & H & G \\
H & T & R & R & H & K & L & R & H & E & H & T & Q & R & H \\
T & G & A & R & G & D & A & R & R & H & G & H & N & K & H \\
L & H & R & M & S & A & A & V & S & E & C & V & G & E & $*$ \\
\hline
\end{tabular}

Figure 2: The amino acid sequence of rLj-RGD3. Histidines are highlighted in yellow; arginines are highlighted in green.

with the control group (set at 100\%; Figure 3). The protective effect of rLj-RGD3 on the PC12 cells subjected to the OGD-R procedure was dose-dependent.

3.3. Effect of rLj-RGD3 on LDH Activity in the Supernatants. As shown in Figure 4, the model group was severely damaged compared with the control group, as indicated by a significant increase in the LDH activity $(p<0.01)$. In contrast, the LDH activity was substantially lower in the rLj-RGD3-treatment groups $(p<0.01)$, and the effect was dose-dependent. However, the LDH activity in the rLj-RGD3 high-dose group showed a less pronounced decrease $(p<0.05)$. The inhibition of $\mathrm{LDH}$ in the edaravone group was not significantly different from that in the rLj-RGD3 high-dose group.

3.4. Effect of rLj-RGD3 on GSH Content in the Cells. Our results (Figure 5) show that, compared with the control group, OGD-R caused a significant decrease in the GSH concentration that was dose-dependently attenuated by the rLj-RGD3 treatment. Significant differences were observed 


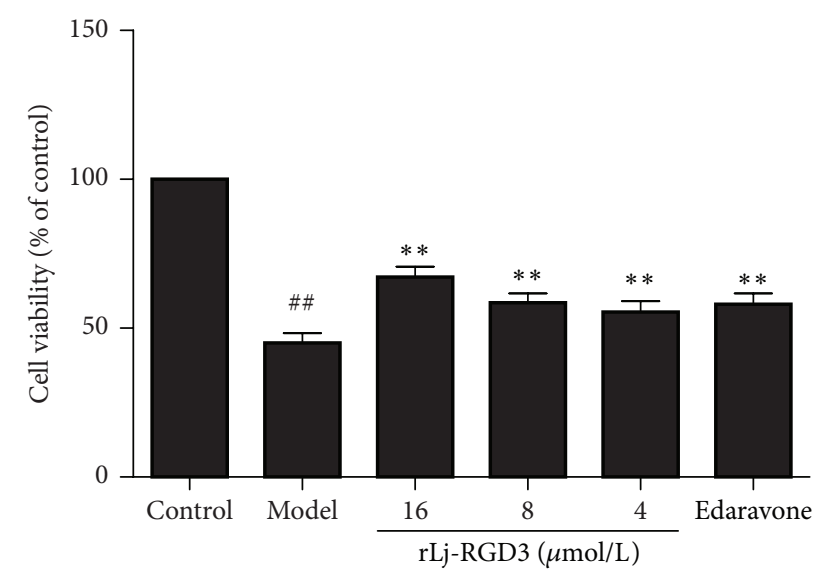

FIGURE 3: The effect of rLj-RGD3 on PC12 cell viability after OGDR-induced injury $(\bar{X} \pm \mathrm{SD}, n=6)$. Normal PC12 cells were subjected to OGD for $2 \mathrm{~h}$ and reperfusion for $24 \mathrm{~h}$. PC12 cell viability was measured using the MTT assay. The control group was set at $100 \%$, and the data obtained in other groups were calculated as the percentage of control. \#\# indicates $p<0.01$ versus the control group; $* *$ indicates $p<0.01$ versus the model group.

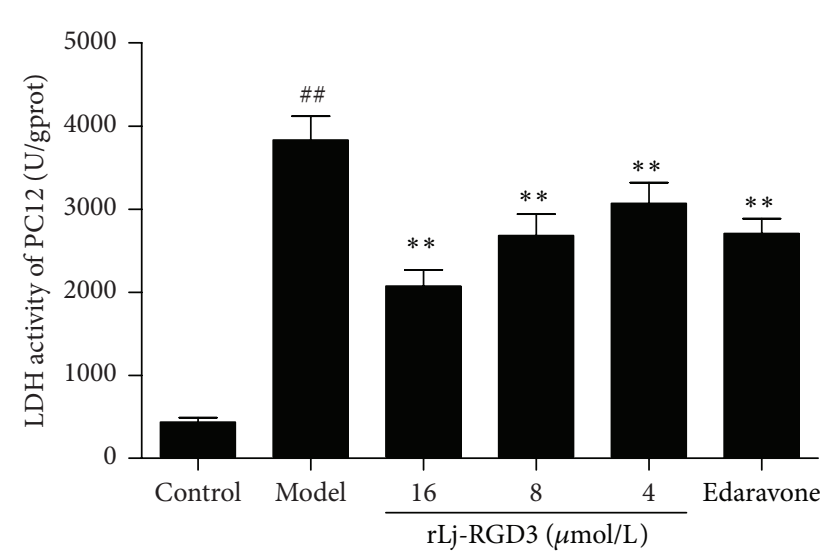

FIgUre 4: The effect of rLj-RGD3 on the LDH activity in the cell supernatants after OGD-R injury to the PC12 cells ( $\bar{X} \pm \mathrm{SD}, n=6)$. The LDH activity in the supernatant fluid was measured using a kit after PC12 cell injury was induced by OGD for $2 \mathrm{~h}$ and reperfusion for $24 \mathrm{~h}$. \#\# indicates $p<0.01$ versus the control group; $* *$ indicates $p<0.01$ versus the model group.

between the model group and the groups pretreated with $\mathrm{rLj}$ RGD3. Furthermore, administration of the high dose of $\mathrm{rLj}^{\mathrm{j}}$ RGD3 significantly increased the content of GSH in the cells compared with those treated with edaravone.

3.5. Morphological Analysis of PC12 Cell Death. To study the morphological aspects of the protective effect of rLj-RGD3 on PC12 cell injury induced by OGD-R, we used AO/EB double-staining fluorescence analysis. Figure 6 depicts the cell apoptosis in each group. The cells of the control group were round with green fluorescence. The most serious injury including orange staining, pyknotic nuclei, decreases in the numbers of adherent cells, and mostly elongated morphology was clearly observed in the model group. The majority of

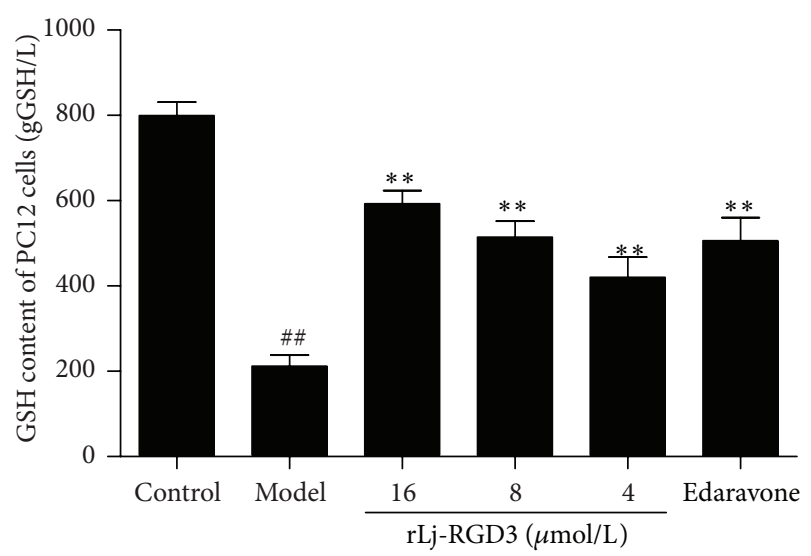

FIGURE 5: The effect of rLj-RGD3 on the GSH content in PC12 cells after OGD-R-induced injury $(\bar{X} \pm \mathrm{SD}, n=6)$. The concentration of GSH in PC12 cells was measured using a kit after injury was induced by OGD for $2 \mathrm{~h}$ and reperfusion for $24 \mathrm{~h}$. \#\# indicates $p<0.01$ versus thecontrol group; $* *$ indicates $p<0.01$ versus the model group.

the cells of the rLj-RGD3 high-dose group exhibited the green fluorescence and the normal structure of living cells. Compared with the model group, apoptosis was significantly inhibited in the treatment groups. Moreover, improvement became more obvious with increasing doses of rLj-RGD3.

3.6. $r L j-R G D 3$ Is Antiapoptotic in PC12 Cells. In the flow cytometric results, the upper left quadrant represents mechanical damage and necrotic cells; the lower left quadrant represents normal cells. The upper right quadrant represents early apoptotic cells and necrotic cells, and the lower right quadrant represents late apoptotic cells. We observed a large number of apoptotic cells in the model group. Figure 7 shows a significant dose-dependent reduction in the early and late apoptotic cells in the rLj-RGD3 treatment groups compared with the model group, particularly for the rLj-RGD3 highdose group. However, there was no significant difference in apoptosis between the rLj-RGD3-treated groups and the edaravone control group.

3.7. FAK and p-FAK Protein Expression. FAK is an important neuroprotective factor downstream from integrins. As shown in Figure 8, expression of both proteins was significantly lower in the model group than in the control group $(p<$ 0.01 ). Treatment with $\mathrm{rLj}$-RGD3 dose-dependently upregulated the expression of these proteins compared with the model group $(p<0.01)$. These results demonstrate that $\mathrm{rLj}$ RGD3 can regulate FAK and p-FAK protein expression and protect the PC12 cells from the damage induced by OGD-R.

3.8. Expression of Caspase- 3 and Bcl-2 Proteins. Caspase- 3 and $\mathrm{Bcl}-2$ are important effectors in the regulation of cell survival or apoptosis. Their expression in the cell directly affects the cell state. The expression levels of both proteins were detected by western blotting. As shown in Figure 9, the expression of Caspase- 3 and Bcl-2 proteins was significantly 


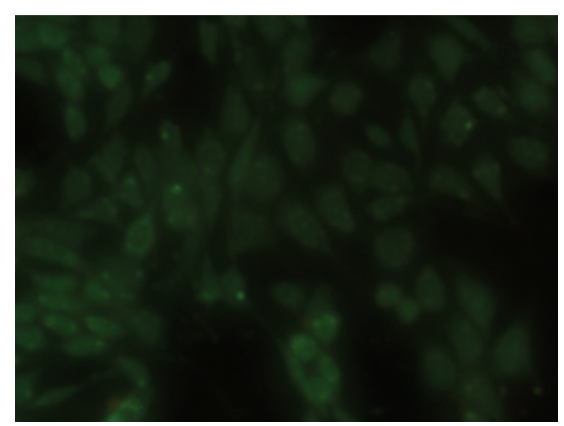

(a)

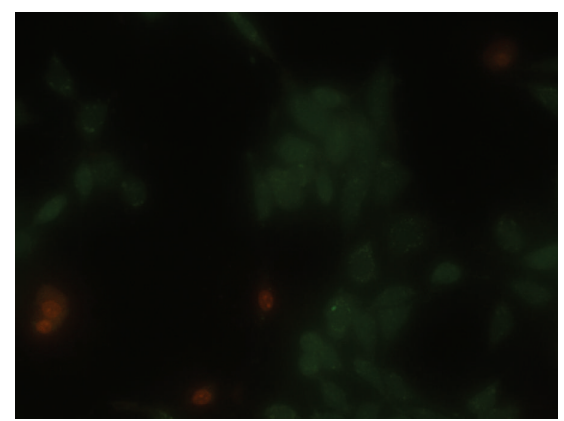

(d)

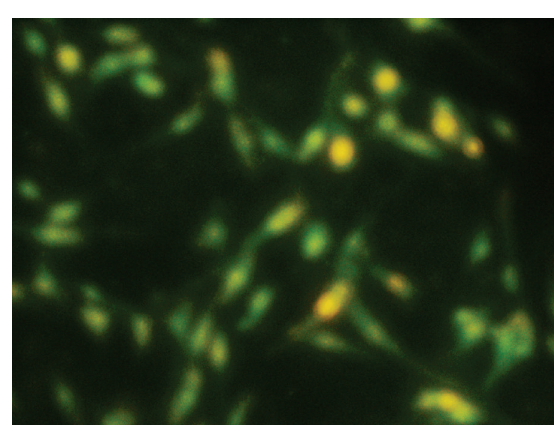

(b)

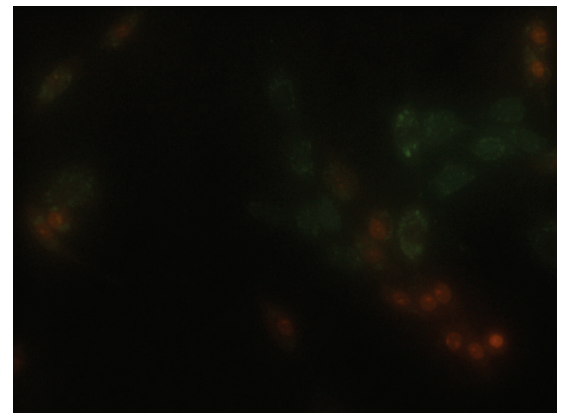

(e)

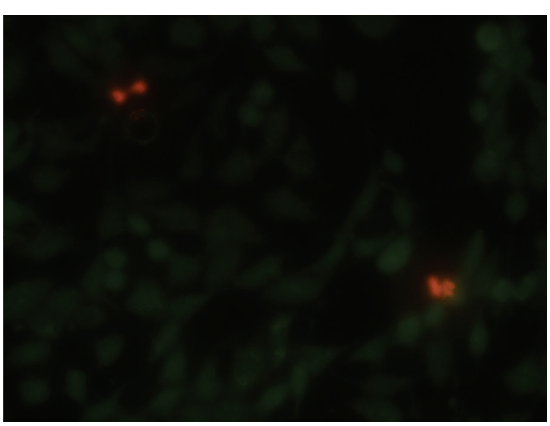

(c)

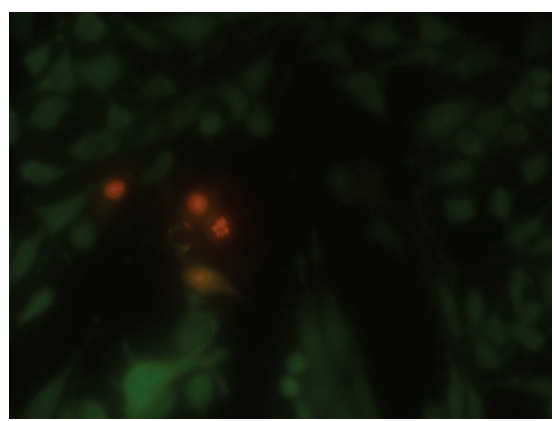

(f)

FIGURE 6: The effect of rLj-RGD3 on PC12 cell apoptosis after OGD-R injury as indicated by AO/EB fluorescence. (a), (b), (c), (d), (e), and (f) represented the control, model, rLj-RGD3 16, 8, and $4 \mu \mathrm{mol} / \mathrm{L}$ dose groups, and edaravone control group, respectively. Most PC12 cells were apoptotic after OGD-R. In contrast, apoptosis of the PC12 cells was significantly reduced in the rLj-RGD3 high-dose group.

increased and inhibited in the model group, respectively. Compared with the model group, Caspase- 3 expression was inhibited significantly, and Bcl-2 expression was increased in a dose-dependent manner in the rLj-RGD3-treated groups.

\section{Discussion}

Ischemic cerebrovascular disease is one of the most common causes of death worldwide [11]. Although an increasing number of studies have described the ischemic mechanisms, including the excessive production of free radicals $[12,13]$, altered calcium homeostasis [14], and N-methyl-D-aspartate excitotoxicity [15], accumulating evidence suggests that the cell death observed during the first few hours of cerebellar ischemia is the result of apoptosis as opposed to necrosis. Necrosis has been considered the predominant form of cerebellar damage generated by ischemia [16]. Moreover, ischemic damage to nerve cells disrupts a series of complex signalling pathways that affect the corresponding biological functions and thus brain function. The terminally differentiated profile of the brain is of particular relevance for cerebellar ischemia [17-19].

$\mathrm{rLj}-\mathrm{RGD} 3$ is a recombinant protein with $3 \mathrm{RGD}$ (Arg-GlyAsp) motifs from the salivary gland secretions of Lampetra japonica, which is a histidine-rich and arginine-rich protein. The deduced amino acid sequence of Lj-RGD3 contains 118 amino acids including 17 histidine and 17 arginine. It is reported that histidine and arginine have cerebral ischemiareperfusion and neuroprotective functions [20,21], so we examined the related activities and mechanism in this paper. Our previous studies about rLj-RGD3 focused on the typical characteristics of RGD-toxin protein, such as platelet aggregation suppression, tumour metastasis, and angiogenesis inhibition [8]; the cerebral ischemia-reperfusion and neuroprotective functions of $\mathrm{rLj}$-RGD3 were reported first in this paper. We examined whether $\mathrm{rLj}$-RGD3 has protective effects on nerve cells through activation of pathways downstream of integrins due to its ability to bind to the specific site on integrins.

In vitro ischemic-like injury is typically induced by OGD in neurons [22]. Rat pheochromocytoma cells (PC12 cells) have become an important tool for in vitro studies of neurology $[23,24]$ because their morphological, physiological, and biochemical functions are similar to those of neurons and because they are easy to use for these types of experiments. PC12 cells are widely used in neurological research in pharmacology and physiology and in in vitro studies of ischemic injury [25]. In this study, we investigated the protective effects of $\mathrm{rLj}-\mathrm{RGD} 3$ using a model of PC12 cell injury induced by OGD-R in vitro and have provided a preliminary discussion of its neuroprotective mechanism.

Lemons and collaborators have found that integrins are involved in the repair of nervous system damage [26]. This study was the basis for studies regarding the rLj-RGD3mediated protection of nerve cells from injury through integrin receptors and provided theoretical support for the present study. Integrins can affect many complex downstream signalling pathways, including FAK, which is important in 


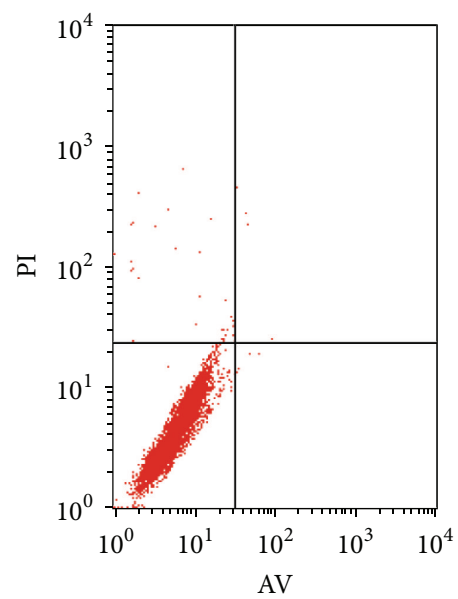

(A)

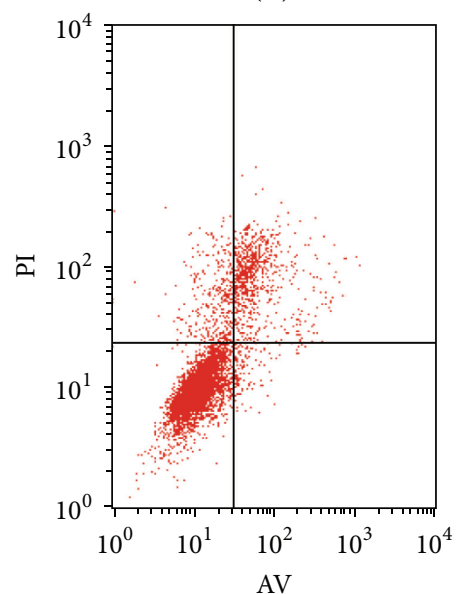

(D)

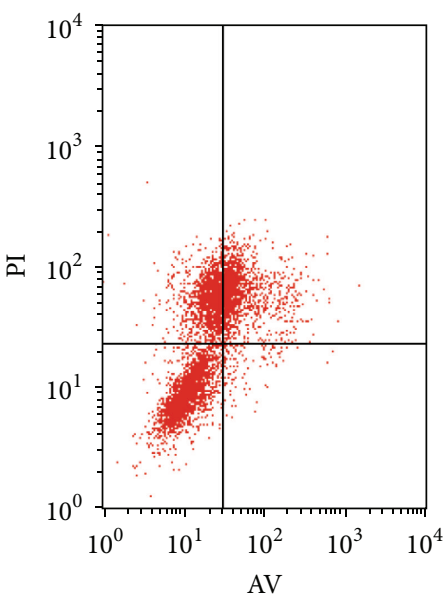

(B)

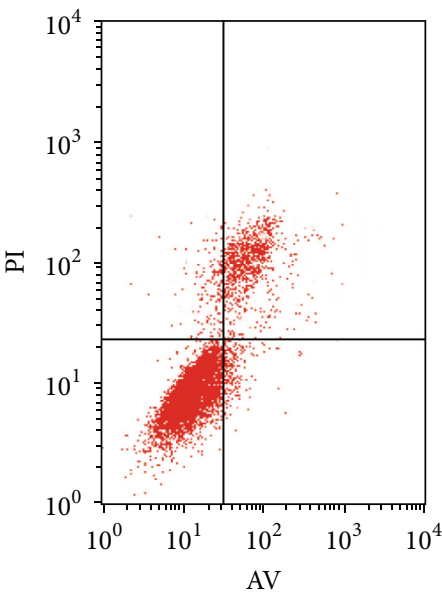

(E)

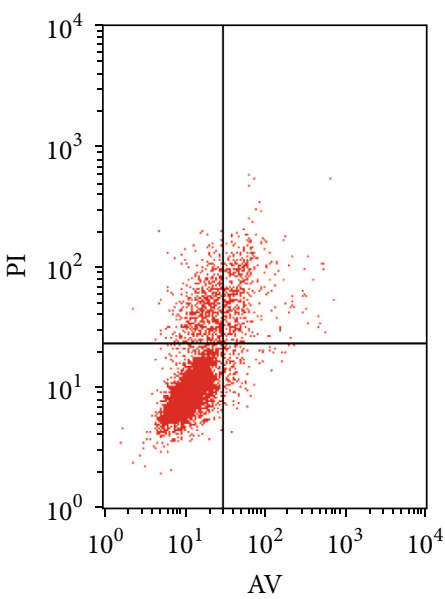

(C)

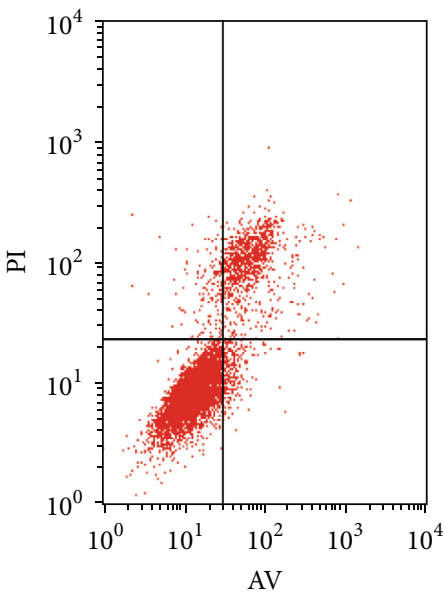

(F)

(a)

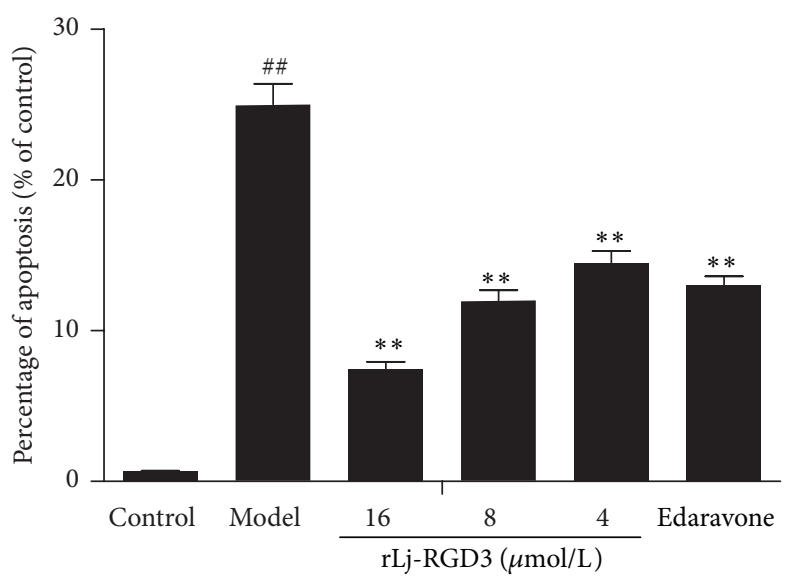

(b)

Figure 7: The effect of rLj-RGD3 on PC12 cell apoptosis after OGD-R injury as indicated by flow cytometry. In Figure 7(a), (A), (B), (C), (D), (E), and (F) represent the control, model, rLj-RGD3 16, 8, and $4 \mu \mathrm{mol} / \mathrm{L}$ dose groups, and the edaravone control group, respectively. The bar graph showed the apoptosis rate in Figure 7(b). The numbers of apoptotic cells in the rLj-RGD3 groups were significantly lower than those of the model group. The results suggested that rLj-RGD3 had an antiapoptotic effect on PC12 cells after OGD-R. The data are shown as means $\pm \mathrm{SD}, n=6$. \#\# indicates $p<0.01$ versus the control group; $* *$ indicates $p<0.01$ versus the model group. 

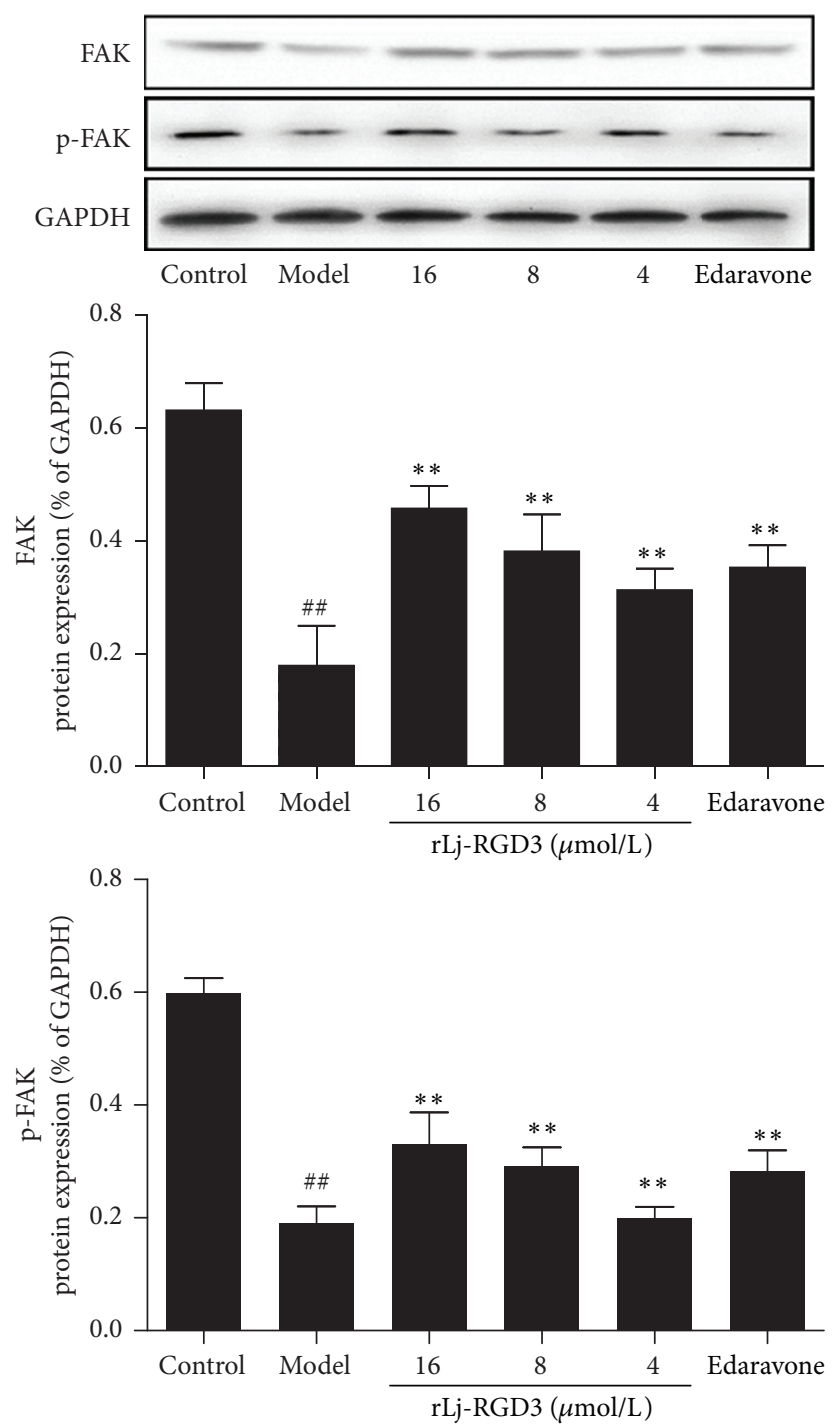

Figure 8: The effect of rLj-RGD3 on the expression of FAK and p-FAK proteins in PC12 cells after OGD-R injury $(\bar{X} \pm \mathrm{SD}, n=3)$. After PC12 cell injury induced by OGD-R, we examined the levels of the proteins of interest, FAK, and its phosphorylated form, by western blotting. The protein bands shown in the figure were quantified after scanning and normalized to GAPDH. The results are presented as the mean \pm SD of three independent experiments. \#\# indicates $p<0.01$ versus the control group; $* *$ indicates $p<0.01$ versus the model group.

the downstream effectors of integrins in the nervous system. Studies have shown that increased levels of integrins on neuronal surfaces can enhance axonal growth. Furthermore, integrin receptors can affect nerve regeneration and functional recovery. Activation of FAK by integrins plays an important role in these processes. In addition, FAK activation can promote neuronal growth, and it has been suggested that FAK integrin signalling pathways play an important role in nerve regeneration $[27,28]$. As indicated by the results of this study, compared with the control group, FAK and p-FAK protein expression were significantly reduced in the OGD$\mathrm{R}$ group. However, rLj-RGD3 induced a dose-dependent increase in FAK and p-FAK protein expression levels, which suggested that rLj-RGD3 can improve the expression of the effectors FAK and promote its activation through activation of the integrin receptors. Therefore, $\mathrm{rLj}$-RGD3 can play a key role in nerve repair and reduce the injury to PC12 cells resulting from OGD-R.

The Caspase family is an important regulator of apoptosis signal factors, and acute neuronal apoptosis is closely associated with the Caspase family. Caspase- 3 is activated only during apoptosis [29]. The results showed that Caspase-3 protein expression was significantly increased in the model group, which is consistent with the discussion above. Additionally, rLj-RGD3 significantly reduced the expression of Caspase-3 in a dose-dependent manner. Our results suggested that $\mathrm{rLj}$ RGD3 significantly inhibited Caspase-3 expression, thereby protecting PC12 cells from OGD-R-induced damage.

$\mathrm{Bcl}-2$ is an important antiapoptotic factor downstream of the activation of the PI3K/Akt pathway. Many studies have demonstrated that it plays a key role in the inhibition of nerve cell apoptosis $[30,31]$. The results show that Bcl-2 

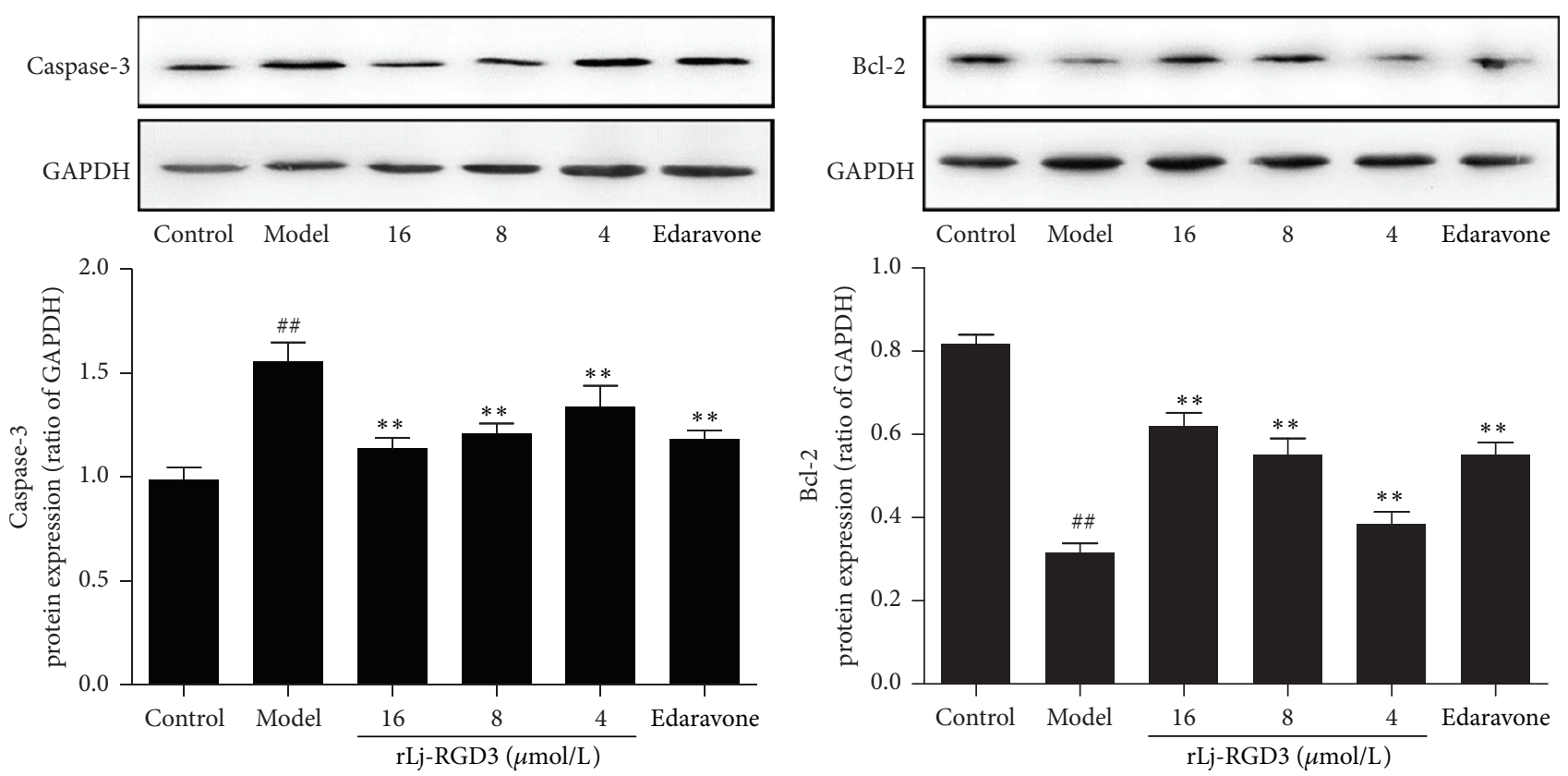

Figure 9: The effect of rLj-RGD3 on the expression of Caspase- 3 and Bcl-2 proteins in PC12 cells after OGD-R injury $(\bar{X} \pm$ SD, $n=3)$. The protein bands shown in the figure were quantified after scanning and normalized to GAPDH. The results are presented as the mean \pm SD of three independent experiments. The results suggested that the studied drug inhibited the apoptosis of PC12 cell subjected to OGD-R. \#\# indicates $p<0.01$ versus the control group; $* *$ indicates $p<0.01$ versus the model group.

protein expression was significantly decreased in the model group, whereas rLj-RGD3 increased $\mathrm{Bcl}-2$ protein expression levels. These results suggested that rLj-RGD3 enhanced antiapoptotic effects and could inhibit brain tissue damage by upregulating $\mathrm{Bcl}-2$ protein expression.

\section{Conclusions}

In summary, this study demonstrated that rLj-RGD3 had obvious protective effects against OGD-R-induced neurotoxicity in PC12 cells. These effects appear to be mediated through integrin receptor-mediated activation of FAK and then through the activation of PI3K/Akt to increase Bcl-2 expression and downregulate Caspase- 3 expression.

\section{Additional Points}

Highlights. A RGD protein from Lampetra japonica (rLjRGD3) blocked OGD-R-induced cytotoxicity. The inhibitory effect of rLj-RGD3 on cytotoxicity was associated with FAK. rLj-RGD3 suppressed the cellular apoptosis induced by OGD-R. Arginine and histidine-rich protein has neuroprotective functions. Our data suggest that $\mathrm{rLj}-\mathrm{RGD} 3$ has a crucial role of neural protection.

\section{Ethical Approval}

The protocol for this research project has been approved by the Institutional Ethics Committee.

\section{Competing Interests}

There are no competing interests for any of the authors related to this paper.

\section{Authors' Contributions}

Li Lv and Qian Lu contributed equally to this work.

\section{Acknowledgments}

This work was supported by National High Technology Research and Development Program ("863" Program, no. 2014AA093502), National Nature Science Foundation of China (no. 81202455 and no. 30770297), Chinese Marine Public Welfare Industry Special Research Project Foundation (no. 201305016), and Major Scientific and Technological Research Projects of Dalian (2014E12SF037).

\section{References}

[1] A. D. Lopez, C. D. Mathers, M. Ezzati, D. T. Jamison, and C. J. Murray, "Global and regional burden of disease and risk factors, 2001: systematic analysis of population health data," The Lancet, vol. 367, no. 9524, pp. 1747-1757, 2006.

[2] H. Yano, Y. Mazaki, K. Kurokawa, S. K. Hanks, M. Matsuda, and H. Sabe, "Roles played by a subset of integrin signaling molecules in cadherin-based cell-cell adhesion," The Journal of Cell Biology, vol. 166, no. 2, pp. 283-295, 2004.

[3] G. E. Morozevich, N. I. Kozlova, M. E. Preobrazhenskaya et al., "The role of $\beta 1$ integrin subfamily in anchorage-dependent 
apoptosis of breast carcinoma cells differing in multidrug resistance," Biochemistry, vol. 71, no. 5, pp. 489-495, 2006.

[4] H. Ohmatsu, T. Kadono, M. Sugaya et al., " $\alpha 4 \beta 7$ Integrin is essential for contact hypersensitivity by regulating migration of T cells to skin," Journal of Allergy and Clinical Immunology, vol. 126, no. 6, pp. 1267-1276, 2010.

[5] U. Hersel, C. Dahmen, and H. Kessler, "RGD modified polymers: biomaterials for stimulated cell adhesion and beyond," Biomaterials, vol. 24, no. 24, pp. 4385-4415, 2003.

[6] S. Verrier, S. Pallu, R. Bareille et al., "Function of linear and cyclic RGD-containing peptides in osteoprogenitor cells adhesion process," Biomaterials, vol. 23, no. 2, pp. 585-596, 2002.

[7] J. E. Sprague, H. Kitaura, W. Zou et al., "Noninvasive imaging of osteoclasts in parathyroid hormone-induced osteolysis using a 64Cu-labeled RGD peptide," Journal of Nuclear Medicine, vol. 48, no. 2, pp. 311-318, 2007.

[8] J. Wang, X. Han, H. Yang et al., "A novel RGD-toxin protein, LjRGD3, from the buccal gland secretion of Lampetra japonica impacts diverse biological activities," Biochimie, vol. 92, no. 10, pp. 1387-1396, 2010.

[9] W. J. Goldberg, R. M. Kadingo, and J. N. Barrett, "Effects of ischemia-like conditions on cultured neurons: protection by low $\mathrm{Na}^{+}$, low $\mathrm{Ca}^{2+}$ solutions," The Journal of Neuroscience, vol. 6, no. 11, pp. 3144-3151, 1986.

[10] P. Han, J.-H. Kang, H.-L. Li et al., "Antiproliferation and apoptosis induced by tamoxifen in human bile duct carcinoma QBC939 cells via upregulated p53 expression," Biochemical and Biophysical Research Communications, vol. 385, no. 2, pp. 251256, 2009.

[11] J.-T. He, J. Mang, C.-L. Mei et al., "Neuroprotective effects of exogenous activin a on oxygen-glucose deprivation in PC12 cells," Molecules, vol. 17, no. 1, pp. 315-327, 2012.

[12] T.-W. Chung, B.-S. Koo, E.-G. Choi, M.-G. Kim, I.-S. Lee, and C.-H. Kim, "Neuroprotective effect of a Chuk-Me-Sun-Dan on neurons from ischemic damage and neuronal cell toxicity," Neurochemical Research, vol. 31, no. 1, pp. 1-9, 2006.

[13] I. Margaill, M. Plotkine, and D. Lerouet, "Antioxidant strategies in the treatment of stroke," Free Radical Biology and Medicine, vol. 39, no. 4, pp. 429-443, 2005.

[14] C. Montell, "The latest waves in calcium signaling," Cell, vol. 122, no. 2, pp. 157-163, 2005.

[15] C. Morin and N. Simon, "Mitochondria: a target for neuroprotective interventions in cerebral ischemia-reperfusion," Current Pharmaceutical Design, vol. 12, no. 6, pp. 739-757, 2006.

[16] S. Paeme, K. T. Moorhead, J. G. Chase et al., "Mathematical multi-scale model of the cardiovascular system including mitral valve dynamics. Application to ischemic mitral insufficiency," BioMedical Engineering Online, vol. 10, article 86, 2011.

[17] O. L. Barbarash, M. V. Zykov, V. V. Kashtalap, and L. S. Barbarash, "Prevalence and clinical significance of multifocal atherosclerosis in patients with ischemic heart disease," Kardiologiya, vol. 51, no. 8, pp. 66-71, 2011.

[18] J. F. Meschia, M. Nalls, M. Matarin et al., "Siblings with ischemic stroke study: results of a genomewide scan for stroke loci," Stroke, vol. 42, pp. 2726-2732, 2011.

[19] G. P. Foster, D. E. Westerdahl, L. A. Foster, J. V. Hsu, and J. D. Anholm, "Ischemic preconditioning of the lower extremity attenuates the normal hypoxic increase in pulmonary artery systolic pressure," Respiratory Physiology and Neurobiology, vol. 179, no. 2-3, pp. 248-253, 2011.
[20] S.-C. Tang, T. V. Arumugam, R. G. Cutler et al., "Neuroprotective actions of a histidine analogue in models of ischemic stroke," Journal of Neurochemistry, vol. 101, no. 3, pp. 729-736, 2007.

[21] F.-Y. Chen and T. J.-F. Lee, "Arginine synthesis from citrulline in perivascular nerves of cerebral artery," Journal of Pharmacology and Experimental Therapeutics, vol. 273, no. 2, pp. 895-901, 1995.

[22] T. Iijima, T. Mishima, K. Akagawa, and Y. Iwao, "Mitochondrial hyperpolarization after transient oxygen-glucose deprivation and subsequent apoptosis in cultured rat hippocampal neurons," Brain Research, vol. 993, no. 1-2, pp. 140-145, 2003.

[23] L. A. Greene and A. S. Tischler, "Establishment of a noradrenergic clonal line of rat adrenal pheochromocytoma cells which respond to nerve growth factor," Proceedings of the National Academy of Sciences, vol. 73, no. 7, pp. 2424-2428, 1976.

[24] H. S. Schubertd, "Cholin2ergic metabolism and synapse formation by a rat nerve cellline," Proceedings of the National Academy of Sciences of the United States of America, vol. 74, no. 6, pp. 2579-25831, 1977.

[25] J. Zhou, Y. Fu, and Xi Can Tang, "Huperzine A protects rat pheochromocytoma cells against oxygen-glucose deprivation," NeuroReport, vol. 12, no. 10, pp. 2073-2077, 2001.

[26] M. L. Lemons and M. L. Condic, "Combined integrin activation and intracellular cAMP cause Rho GTPase dependent growth cone collapse on laminin-1," Experimental Neurology, vol. 202, no. 2, pp. 324-335, 2006.

[27] U. Schlomann, J. C. Schwamborn, M. Müller, R. Fässler, and A. W. Püschel, "The stimulation of dendrite growth by Sema3A requires integrin engagement and focal adhesion kinase," Journal of Cell Science, vol. 122, no. 12, pp. 2034-2042, 2009.

[28] M. L. Lemons and M. L. Condic, "Integrin signaling is integral to regeneration," Experimental Neurology, vol. 209, no. 2, pp. 343-352, 2008.

[29] S. Namura, J. Zhu, K. Fink et al., "Activation and cleavage of caspase-3 in apoptosis induced by experimental cerebral ischemia," The Journal of Neuroscience, vol. 18, no. 10, pp. 36593668, 1998.

[30] J. Chen, S. H. Graham, M. Nakayama et al., "Apoptosis repressor genes $\mathrm{Bcl}-2$ and $\mathrm{Bcl}-\mathrm{x}$-long are expressed in the rat brain following global ischemia," Journal of Cerebral Blood Flow and Metabolism, vol. 17, no. 1, pp. 2-10, 1997.

[31] M. S. Lawrence, D. Y. Ho, G. H. Sun, G. K. Steinberg, and R. M. Sapolsky, "Overexpression of Bcl-2 with herpes simplex virus vectors protects CNS neurons against neurological insults in vitro and in vivo," Journal of Neuroscience, vol. 16, no. 2, pp. 486496, 1996. 

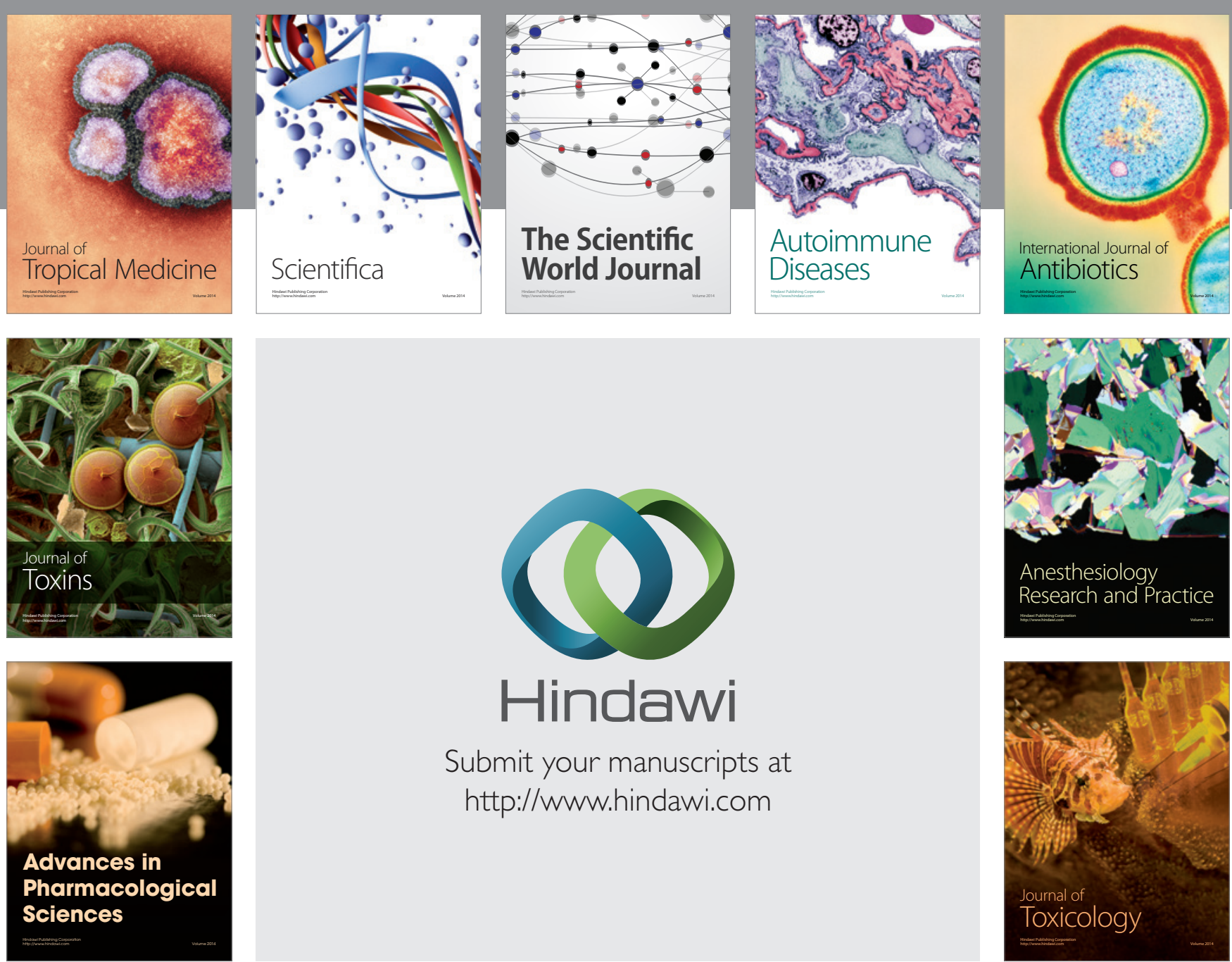

\section{Hindawi}

Submit your manuscripts at

http://www.hindawi.com
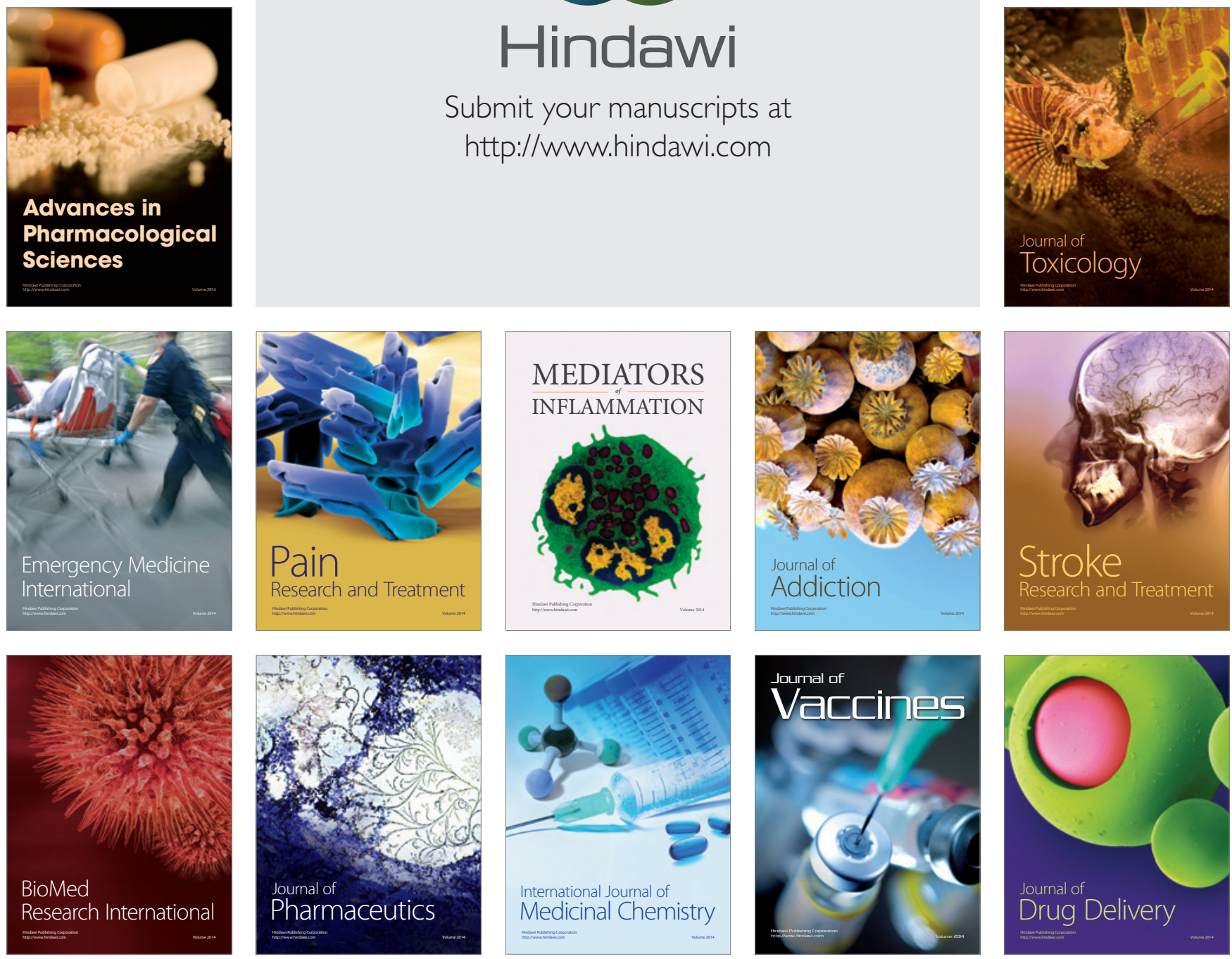